\title{
Variabilidad genética para la producción de toxina en poblaciones de Microcystis aeruginosa en dos embalses de abastecimiento de Andalucía
}

\author{
A. Martín ${ }^{1}$, E. Carrillo y E. Costas ${ }^{2}$ \\ ${ }^{1}$ Empresa Municipal de Abastecimiento y Saneamiento de Aguas de Sevilla, S A. Estación de Ecología \\ Acuática "Príncipe Alberto I de Mónaco". Avda. Leonardo da Vinci s/n. 41092 Sevilla. \\ ${ }^{2}$ Laboratorio de Genética de Microalgas. Facultad de Veterinaria. Universidad Complutense 28040, Madrid.
}

\section{RESUMEN}

Las proliferaciones de cianobacterias tóxicas ocurren a menudo en aguas continentales. Entre todas las especies de cianobacterias, la especie productora de microcistinas (hepatotoxinas) Microcystis aeruginosa, es la principal fuente de problemas tanto para animales como para el hombre. Las proliferaciones de Microcystis se caracterizan por presentar una fuerte heterogeneidad en su producción de toxinas tanto espacial como temporalmente. Habitualmente se atribuye a factores ambientales esta heterogeneidad. Sin embargo, las diferencias genéticas podrían jugar un papel destacado. En este trabajo hemos detectado y cuantificado variabilidad genética para la producción de toxina tanto dentro de una proliferación como cuando se encuentran a baja concentración en dos poblaciones de $M$. aeruginosa. Para ello se aislaron y cultivaron 17 cepas de $M$. aeruginosa de dos embalses de Andalucía, y se mantuvieron en idénticas condiciones ambientales. La producción de microcistinas se midió utilizando un test ELISA en cultivos completamente aclimatados. Se detectó variabilidad estadísticamente significativa entre las distintas cepas. En contraste con el punto de vista clásico, la mayoría de esta variabilidad para producción de toxinas es debida a diferencias genéticas entre especies. Este hecho introduce una fuente de incertidumbre en los sistemas de alerta temprana de embalses de abastecimiento basados generalmente en recuentos de abundancia celular

Palabras clave: variabilidad genética, producción de toxinas, heredabilidad, Microcystis aeruginosa.

\begin{abstract}
Toxic cyanobacteria blooms often occur worldwide in freshwater environments. From all cyanobacteria toxic species, Microcystis aeruginosa, hepatotoxin (microcystin) producing, is known to be the main source of noxious outbreaks to humans and animals. These outbreaks are characterized by the occurrence of Microcystis blooms, which show a high degree of heterogeneity in toxin production in both spatial and temporal scales. Usually, this heterogeneity is explained by environmental factors. However, genetic differences can play an important role. In this work, genetic variability for toxin production was detected within and among natural populations of M. aeruginosa. Seventeen clonal cultures of M. aeruginosa, isolated from two water supply reservoirs of Andalusia (S-Spain) were grown under the same environmental conditions. Toxin quota was measured in fully acclimated balanced growth cultures using a commercial ELISA test kit. Statistically significant variability for the production of microcystin was found among the different strains. Most of intra-bloom and inter-bloom variability in toxin production is due to genetic components in contrast with classical environmental point of view. This fact introduces an important source of uncertainty in early-warning systems of water supply management based on cell abundance.
\end{abstract}

Keywords: genetic variability, toxin production, heritability, Microcystis aeruginosa.

\section{INTRODUCCIÓN}

Las cianobacterias tóxicas proliferan en aguas continentales de todo el mundo, en especial cuando tienen un alto grado de eutrofía, consti- tuyendo una importante preocupación a nivel internacional (Bartram et al., 1999). Unas 40 especies son capaces de producir toxinas de diversos tipos, causantes de mortandades masivas de animales (Carmichael, 2001, Alonso- 
Andicoberri et al., 2002), que incluso pueden matar a seres humanos (Jochimsen et al., 1998).

La especie que con mas frecuencia aparece implicada en episodios tóxicos es Microcystis aeruginosa (Kützing) Kützing, que produce hepatotoxinas (microcistinas), y prolifera hasta producir espesas natas superficiales en embalses y lagos (Carmichael \& Schwartz 1984, Sivonen 1990, Skulberg et al., 1993). Sin embargo, no todos las grandes proliferaciones (blooms) de Microcystis son tóxicas, y en ocasiones la toxicidad de una misma proliferación varía con el tiempo, e incluso pueden observarse manchas de elevada y baja toxicidad alternando simultáneamente en un mismo embalse (Benndorf \& Henning 1989, Codd et al., 1989, Vezie et al., 1998, Kabernick \& Neilan 2001).

Esta heterogeneidad para la producción de toxinas suele ser atribuida a factores ambientales, pues los estudios de laboratorio han demostrado que la producción de microcistina varía en función de la concentración de nitrógeno y fósforo, de la irradiancia, y de la temperatura (Kotak et al., 2000, Kaebernick \& Neilan, 2001). En general la máxima producción de toxina suele darse en condiciones óptimas de crecimiento (Sivonen \& Jones, 1999, Kaebernick \& Neilan, 2001).

Sin embargo, los componentes genéticos también parecen jugar un importante papel en estas variaciones en la producción de toxina (Jacoby et al., 2000, Kaebernick \& Neilan, 2001, Carrillo et al., 2003). Los estudios de laboratorio han demostrado que los factores ambientales inducen cambios en la concentración de toxinas, pero de menor magnitud que los observados entre diferentes cepas cultivadas en las mismas condiciones ambientales (Sivonen \& Jones, 1999). Dado que se han aislado numerosos clones de Microcystis con distinto grado de toxicidad (Alvarez et al., 1998, Martín et a1., 2000, Carrillo et al., 2003), puede pensarse que parte de la variación en la concentración de toxina de una misma proliferación puede ser debida a cambios en las frecuencias relativas de genotipos con distinto grado de toxicidad.

Consecuentemente la caracterización de la variabilidad genética para toxicidad en las poblaciones de Microcystis podría ser útil para la gestión de embalses destinados al abastecimiento de poblaciones. Así, numerosos autores han intentado estudiar la genética de la producción de toxinas a nivel molecular (Tillet et al., 2000, Kabernick et al., 2000, Kabernick \& Neilan 2001, Bitencourt-Oliveira et al., 2001, Kurmayer et al., 2002, Bitencourt-Oliveira 2003). El uso de procedimientos moleculares es sin duda una aproximación prometedora. Tales estudios asumen que los genes operan en un sistema cerrado en el cual la presencia o ausencia de una secuencia génica o un producto génico determina directamente el genotipo de un individuo. Un punto de vista alternativo asume que la mayoría de los genes y productos génicos actúan en el medio interno de las células donde se producen complejas interacciones relacionadas con los efectos del medio externo. Consecuentemente, el fenotipo resultante es a menudo modificado a través de la interacción entre un genotipo particular y el medio ambiente interno y externo (Falconer 1986, Klug \& Cummings 1997). Tales interacciones sutiles dificultan el entendimiento de la tan debatida polémica acerca de la importancia relativa de genes y ambiente. En este sentido, el uso de los procedimientos de genética cuantitativa pueden ser una alternativa interesante para analizar la variabilidad en la producción de toxinas.

Recientemente nuestro grupo comenzó a aplicar técnicas de genética cuantitativa al estudio de la variabilidad genética en para la producción de microcistinas (Carrillo et al., 2003). La producción de microcistinas es un carácter cuantitativo, en el que su expresión fenotípica no siempre es un reflejo directo del genotipo, pero dado que esta producción puede medirse detalladamente en diferentes cepas de $M$. aeruginosa aisladas de poblaciones naturales, los experimentos con numerosas cepas sirven para analizar las causas de la variación en la producción de microcistinas (Carrillo et al., 2003). Además, puede calcularse la proporción de la variabilidad observada para la producción de microcistinas que se 
Tabla 1. Referencias de las cepas de Microcystis aeruginosa aisladas en los embalses estudiados. Different clones from isolated Microcystis aeruginosa of the water supply reservoirs.

\begin{tabular}{cccc}
\hline Área & Fecha de aislamiento & No de cepas & Designación \\
\hline La Minilla reservoir & Dec. 1999 & 11 & de Ma1M a Ma11M \\
\hline \multirow{3}{*}{ El Gergal reservoir } & Apr. 1999 & 4 & de Ma2G a Ma5G \\
& Jun. 1999 & 1 & Ma6G \\
& May 2000 & 1 & Ma7G \\
\hline
\end{tabular}

debe a las diferencias genéticas o a las diferencias ambientales. En este trabajo se analiza la variabilidad genética para la producción de microcistinas en poblaciones de Microcystis aeruginosa procedentes de dos embalses de abastecimiento de Andalucía.

\section{MATERIAL Y MÉTODOS}

Se aislaron 17 cepas de Microcystis aeruginosa procedentes de dos embalses Andaluces (Tabla 1). Las cepas de uno de los embalses (cepas MalM a Ma11M) se aislaron de la misma muestra de agua de 1 litro recogida durante una misma proliferación mientras que las del otro al embalse (cepas Ma2G a Ma7G) se aislaron de poblaciones Microcystis a baja densidad en tres fechas diferentes. Cada cepa procedía de una única célula aislada de una sola colonia mediante un micromanipulador-microinyector. Todas las cepas se cultivaron en idénticas condiciones ambientales, en frascos de cultivo con $20 \mathrm{ml}$ de medio de cultivo BG11, a $25^{\circ} \mathrm{C}$ y en luz continua de $250 \mu \mathrm{mol}$ fotones $\mathrm{m}^{-2} \mathrm{~s}^{-1}$ procedente de tubos fluorescentes day-light. Las cepas se mantuvieron en crecimiento exponencial y asincrónico ('balanced growth') mediante transferencias seriadas de un inóculo a medio nuevo, y aclimatadas a las condiciones ambientales del experimento. Los detalles acerca del aislamiento, cultivo, ciclo, evidencias de monoclonalidad de las cepas, etc., se dan en López-Rodas \& Costas (1997).

La toxicidad de cada uno de los clones se midió usando un test ELISA específico para microcistinas (EnviroGard Microcystin QuantiTube Test Kit, de Strategic Diagnostic INC.), siguiendo las recomendaciones del fabricante. Este kit mide microcistinas, y a pesar de que no distingue entre tipos de toxinas, esto no es relevante en nuestro trabajo ya que se pretendía medir la variabilidad para la producción total de toxina. Recientemente hemos publicado los detalles acerca del procedimiento de extracción y medida de toxinas y clorofila en Microcystis (Carrillo et al., 2003). La concentración de toxina se expresa como mg de microcistina-LR equivalente por $\mathrm{mg}$ de clorofila $a$ de acuerdo a las recomendaciones de Sivonen \& Jones, (1999). Una discusión detallada acerca de la calidad del método de EnviroGard Microcystin QuantiTube Test Kit para medir microcistinas puede verse en Carrillo et al., (2003). La fiabilidad, reproducibilidad y precisión de las medidas de microcistinas fueron respectivamente $98 \%, 96 \%$ y 1.2 ppb (para detalles ver Carrillo et al., 2003).

La heredabilidad (h2), fracción de varianza genética respecto a la varianza fenotípica, se calculó mediante un ANOVA jerárquico según el siguiente modelo:

$$
\mathrm{y}=\mu+\mathrm{S}+\mathrm{R}(\mathrm{S})+\mathrm{e}
$$

donde:

$$
\begin{aligned}
& \mathrm{y}=\text { valor del carácter } \\
& \mu=\text { media estándar } \\
& \mathrm{S}=\text { cepa } \\
& \mathrm{R}(\mathrm{S})=\text { replicados de las cepas } \\
& \mathrm{E}=\text { valor residual }
\end{aligned}
$$




\section{RESULTADOS Y DISCUSIÓN}

La concentración media de microcistina (con un intervalo de confianza del 95\%) para cada cepa se muestra en la figura 1, donde puede apreciarse la variabilidad en la concentración de toxina entre las diferentes cepas. El clon más tóxico resultó Ma6G (1.25 $\mu \mathrm{g}$ de microcistina-LR equivalente por mg de clorofila $a$ ). Alguna de las cepas estudiadas muestra una alta concentración de toxinas $(0.5 \mathrm{a} 1 \mu \mathrm{g}$ de microcistina-LR equivalente por mg de clorofila $a$ ). La mayoría de los clones producen una concentración de toxina moderada (menos de $0.5 \mu \mathrm{g}$ de microcistina-LR equivalente por $\mathrm{mg}$ de clorofila $a$ ) y sólo dos clones podrían considerarse como no tóxicos (Ma9M y Ma2G).

Se encontró una variabilidad estadísticamente significativa en la producción de microcistinas en las poblaciones procedentes de ambos embalses ( $\mathrm{p}<0.001$, ANOVA test). Dado que la concentración de microcistinas se midió en las mismas condiciones ambientales, esta variabilidad refleja las diferencias genéticas existentes entre las cepas. Un estimador de la variabilidad genética encontrada entre cepas es el coeficiente de variación. Entre las cepas procedentes de la misma proliferación de un embalse encontramos un $\mathrm{CV}$ $=47.4 \%$, mientras que entre cepas aisladas en el otro embalse a lo largo de un año, encontramos un $\mathrm{CV}=106.1 \%$. En ambos casos la variabilidad genética encontrada es elevada, pero nos parece especialmente relevante destacar la variabilidad genética encontrada dentro de una misma proliferación. Este hecho sugiere que las diferencias genéticas constituyen la principal causa de esta variabilidad. En concreto hemos obtenido una heredabilidad de 0.75 procesando conjuntamente el global de las cepas de los dos embalses. Esto indica que el $75 \%$ de la variabilidad observada para la producción de toxinas es debida a causas genéticas. Este valor parece suficientemente elevado como para asumir que gran parte de la heterogeneidad para la producción de microcistina detectada en las proliferaciones, lejos de ser debida exclusivamente a factores ambientales, es un problema en el que juega un papel importante el componente genético. Anteriormente, en estos embalses se habían identificación diferentes serotipos de Microcystis aeruginosa con distinto grado de toxicidad (Martín et al., 2000). Desafortunadamente, este hecho introduce un cierto grado de incertidumbre a la hora de aplicar sistemas de alerta temprana en aguas de abasteci-

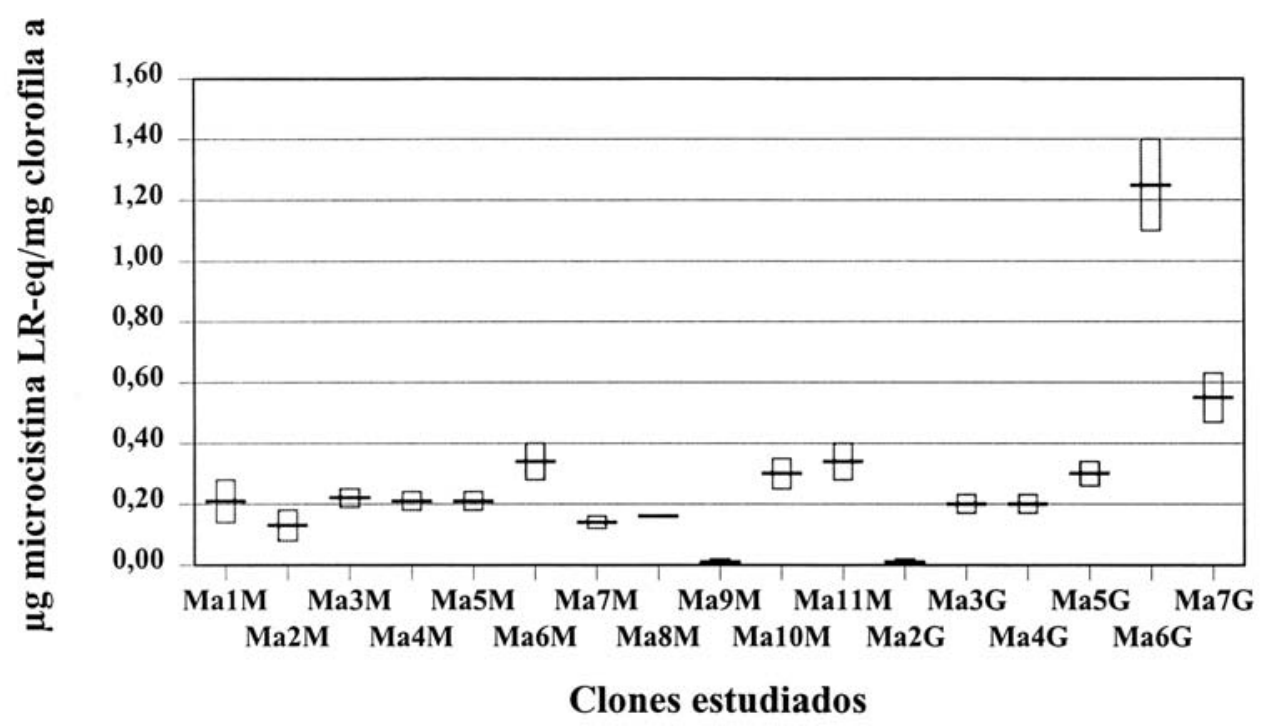

Figura 1. Concentración media de microcistina-LR equivalente e intervalos de confianza (95\%) encontrados en los diferentes clones. Mean microcystin-LR equivalent concentrations of differents clones. Confidence intervals (95\%) are indicated by vertical lines. 
miento basados exclusivamente en procedimientos de abundancia celular.

Se observó además que la mayoría de las cepas estudiadas producen una concentración de toxina moderada, mientras que sólo unas pocas no son tóxicas. Esto reabre el debate sobre el papel que desempeña la producción de toxinas en la ecología de las cianobacterias. Aunque se conoce tanto de la estructura como de los efectos de las microcistinas, apenas existen hipótesis sobre el papel ecológico que desempeñan estas toxinas. De forma tentativa, nuestros resultados apuntan a que la producción de toxina no está sujeta a fuerte selección natural, pues en general los caracteres bajo fuerte efecto de la selección suelen presentar bajas heredabilidades.

\section{CONCLUSIONES}

La variabilidad genética para la producción de microcistinas encontrada en las poblaciones de Microcystis, es considerable, incluso dentro de un misma proliferación.

Los sistemas de alerta temprana en aguas de abastecimiento basados en recuentos celulares no son eficaces, ya que la variabilidad genética introduce incertidumbre.

La producción de microcistinas no parece estar sujeta a fuerte selección natural.

\section{AGRADECIMIENTOS}

Este trabajo ha sido financiado, en parte, por el Proyecto REN 2000-0771/HID. Así mismo, agradecemos a EMASESA las aportaciones económicas realizadas y los distintos recursos facilitados. Finalmente, uno los autores (E. Carrillo) es beneficiario de una beca predoctoral de F.P.I. de la Comunidad de Madrid.

\section{BIBLIOGRAFÍA}

ALONSO-ANDICOBERRY, C., L. GARCÍAVILLADA, V. LÓPEZ-RODAS, \& E. COSTAS.
2002. Catastrophic mortality of flamingos in a Spanish national park caused by cyanobacteria. Veterinary Record, 151: 706-708.

ÁLVAREZ M. J., A. BASANTA, V. LÓPEZ-RODAS \& E. COSTAS. 1998. Identification of different serotypes during a Microcystis aeruginosa bloom in a SW Spanish reservoir. In: Harmful Algae. B. Reguera, J. Blanco, M.L. Fernández \& T. Wyatt (eds.): 291-295. Xunta de Galicia and Intergovernmental Oceanographic Commission of UNESCO, Paris, France.

BARTRAM J., W. W. CARMICHAEL, I. CHORUS, G. JONES \& O. M. SKULBERG. 1999. Introduction. In: Toxic cyanobacteria in water. A guide to their public health consequences, monitoring and management. I. Chorus \& J. Bartram (eds.): 1-14. Routledge, London.

BENNDORF J. \& M. HENNING. 1989. Daphnia and toxic blooms of Microcystis aeruginosa in Bautzen Reservoir. Internationale Revue der gesamten Hydrobiologie, 74: 233-248.

BITTENCOURT-OLIVEIRA, M. C. 2003. Detection of potential microcystin-producing cyanobacteria in Brazilian reservoirs with a $m c y \mathrm{~B}$ molecular marker. Harmful Algae, 2: 51-60.

BITTENCOURT-OLIVEIRA, M. C., M. CABRAL OLIVEIRA \& C. J. S. BOLCH. 2001. Genetic variability of Brazilian strains of the Microcystis aeruginosa complex (Cyanobacteria / Cyanophyceae) using the phycocyanin intergenic spacer and flanking regions (cpcBA). J. Phycol., 37: 810-818.

CARMICHAEL, W. W. 2001. Health effects of toxin-producing cyanobacteria: "The Cyano HABs". Hum. Ecol. Risk. Assess., 7: 1393-1407.

CARMICHAEL W. W. \& L. D. SCHWARTZ. 1984. Preventing livestock deaths from blue-green poisoning. Farmers Bulletin 2275. US Dept of Agriculture, Washington, D.C.

CARRILLO, E., L. M. FERRERO, C. ALONSOANDICOBERRY, A. BASANTA, A. MARTÍN, V. LÓPEZ-RODAS \& E. COSTAS. 2003 Interstrain variability in toxin production in the populations of the cyanobacterium Microcystis aeruginosa from water-supply reservoirs of Andalusia and lagoons of Doñana National Park (southern Spain). Phycologia, 42: 269-274.

CODD, G. A., W. P. BROOKS, I. M. PRIESTLEY, G. K. POON \& S. G. BELL. 1989. Production, detection and quantification of cyanobacterial toxins. Toxicity Assessment, 4: 499-511. 
LÓPEZ RODAS, V. \& E. COSTAS 1997. Characterization of morphospecies and strains of Microcystis (Cyanobacteria) from natural populations and laboratory clones using cell probes (lectins and antibodies). J. Phycol., 33: 446-454

FALCONER, D. S. 1989. Introducción a la genética cuantitativa. México D.F.: CECSA.

JACOBY, J. M., D. C. COLLIER, E. B. WELCH, F. J. HARDY \& M. CRAYTON. 2000. Environmental factors associated with a toxic bloom of Microcystis aeruginosa. Can. J. Fish. Aq. Sci., 57: 231-240.

JOCHIMSEM, E. M., W. W. CARMICHAEL, L. AN, J., CARDO, D. M., COOKSON, S. T., HOLMES, C. E. M., ANTUNES, M. B., MELO, D. A. DE M., LYRA, T. M., BARRETO, V. S. T., AZEVEDO, S. M. . \& JARVIS, W. R. 1998. Liver failure and death after exposure to microcystins at a hemodialysis center in Brazil. N. Engl. J. Med., 338: 873-878.

KAEBERNICK, M. \& B. A. NEILAN. 2001. Ecological and molecular investigations of cyanotoxin production. FEMS Microbiol. Ecol., 35: 1-9.

KAEBERNICK M., B. A. NEILAN, T. BORNER \& E. DITTMANN. 2000. Light and the transcriptional response of the microcystin biosynthesis gene cluster. Appl. Environ. Microbiol., 66: 3387-3392.

KOTAK B. G., A. K. Y. LAM, E. E. PREPAS \& S. E. HRUDEY. 2000. Role of chemical and physical variables in regulating microcystin-LR concentration in phytoplankton of eutrophic lakes. Can. J. Fish. Aq. Sci., 57: 1584-1593.

KLUG W. S. \& M. R. CUMMINGS. 1997. Concepts of genetics, $5^{\text {th }}$ ed. Prentice Hall, New Jersey. 703 pp.

KURMAYER, R., E. DITTMANN, J. FASTNER \& I. CHORUS. 2002. Diversity of microcystin genes within a population of the toxic cyanobacterium Microcystis spp. in lake Wannsee (Berlin, Germany). Microb. Ecol., 43: 107-118

MARTIN, A., E. CARRILO, E. COSTAS \& A. BASANTA. 2000. Identificación de serotipos de Microcystis aeruginosa con distinto grado de toxicidad en un embalse de abastecimiento. Tecnología del Agua, 199: 54-59.

SIVONEN, K. 1990. Effects of light, temperature, nitrate, orthophosphate and bacteria on growth of and hepatotoxin production by Oscillatoria agardhii strains. Appl. Environ. Microbiol., 56: 2658-2666.

SIVONEN, K. \& G. JONES. 1999. Cyanobacterial toxins. In: Toxic Cyanobacteria in water. A guide to their public health consequences, monitoring and management. I. Chorus y J. Bartram (eds): 41-111. Routledge, London.

SKULBERG, O. M., W. W. CARMICHAEL, G. A. CODD \& R. SKULBERG. 1993. Toxigenic cyanophytes - identification and taxonomy. In: Algal toxins in seafood and drinking water. I. R. Falconer (ed.): 145-164. Academic Press, London.

TILLETT, D., E. DITTMAN, M. ERHARD, H. VON DOREN, T. BÖRNER \& B.A. NEILAN. 2000. Structural organization of microcystin biosynthesis in Microcystis aeruginosa PCC 7806: an integrated peptide-polyketide synthetase system. Chemistry and Biology, 7: 753-764.

VEZIE, C., L. BRIENT, K. SIVONEN, G. BERTRU, J. C. LEFEUVRE \& M. SALKINOJA-SALONEN. 1998. Variation of microcystin content of cyanobacterial blooms and isolated strains in Lake Grand Lieu (France). Microb. Ecol., 35: 126-135. 